\title{
Urbanisation and global environmental change: new intergenerational challenges
}

\author{
David Simon \\ Department of Geography \\ Royal Holloway \\ University of London \\ EGHAM, Surrey TW20 0EX, UK \\ E-mail: d.simon@rhul.ac.uk
}

\begin{abstract}
The recent Stern Review Report into climate change has focused attention on the economics of Global Environmental Change (GEC), producing arguably the most authoritative review to date. The Fourth Assessment Report of the Intergovernmental Panel on Climate Change (IPCC), due for publication in spring 2007, will underpin yet further the growing scientific consensus regarding the seriousness of GEC and the urgency of serious measures to tackle its effects. This paper outlines the particular - and still weakly understood - bidirectional relationships between urbanisation and GEC, highlighting the new research needs and particular issues of intergenerational equity that arise.
\end{abstract}

Keywords: urbanisation; Global Environmental Change; GEC; sustainability; intergenerational equity.

Reference to this paper should be made as follows: Simon, D. (2007) 'Urbanisation and global environmental change: new intergenerational challenges', Int. J. Green Economics, Vol. 1, Nos. 3/4, pp.299-306.

Biographical notes: David Simon is Professor of Development Geography at Royal Holloway, University of London, where he heads the Centre for Developing Areas Research (CEDAR). He is a founding member of the Scientific Steering Committee of the Urbanisation and Global Environmental Change (UGEC) 'core project' of the International Human Dimensions Programme on Global Environmental Change (IHDP) as well as co-convenor of the new UK National Committee on the Human Dimensions of GEC. He has published widely on diverse theoretical, practical and policy-related issues of development and the environment, most recently (as editor) Fifty Key Thinkers on Development (Routledge, 2006) and (as co-editor) The Peri-Urban Interface; Approaches to Sustainable Natural and Human Resource Use (Earthscan, 2006). He is also Joint Editor of the Journal of Southern African Studies.

\section{Introduction}

Global Environmental Change (GEC) has necessarily gained great prominence in recent years, also rising up the political agenda. Rather like sustainable development in the early 1990s, it has become a virtual buzzword and in some green quarters even caused a moral panic. As with all such topical agendas, however, increased popularisation does not guarantee that the concept and issues are adequately understood. This article therefore 
seeks to address this need. It defines and explains GEC against a broad context of increasing scientific and political concern, and then explores the far less well understood nature of the bidirectional linkages between urbanisation and GEC. The associated issues of intergenerational equity are addressed in that light.

\subsection{Global environmental change}

Global environmental changes are most simply defined as those that alter Earth system functioning and [are] experienced globally (e.g., climate change) and those that occur in specific areas and the aggregate effects of which contribute to global change (e.g., land conversion) (Vitousek, 1992). Numerous definitional variations exist, reflecting in part the disciplinary backgrounds of their originators and their views on the relative contributions of natural versus anthropogenic causes to GEC. Rather than debate their relative merits, however, it is more useful to underscore the fact - borne out by the Stern Review on the Economics of Climate Change (2006) - that the consensus coalescing over recent years around the importance of anthropogenic causes is now overwhelming. This is not to suggest that long-term climate change and climatic variations have not been an enduring feature of the Earth's history; rather that the nature and rate of change are now such that 'natural' cyclical explanations are inadequate.

A common misperception is that GECs differ little from disasters and therefore that the considerable literature accumulated on that subject, including prediction, coping and recovery strategies, since the 1980s, can be applied directly. While there is indeed some overlap, there are also major differences. Apart from droughts, most supposedly 'natural' disasters are sudden, one-off events like volcanic eruptions, earthquakes and tsunamis, hurricanes or one-in-a-thousand-year floods, even if science is gradually improving its predictive skills and thus enabling the establishment of early warning systems. These events are also usually of short duration: a few minutes, hours or days. The central policy issues therefore relate to providing early warning, undertaking timely (usually temporary) evacuation and hastening effective post-disaster reconstruction.

By contrast, GEC involves far more varied and long-term changes. The two essential distinguishing points about GEC are that it is contributing substantially to the increasing frequency and severity of extreme events and disasters, and that these increases are occurring on top of a long-term 'secular' rise in sea level and atmospheric $\mathrm{CO}_{2}$ concentrations. Some of the most dramatic changes are slow-onset events but once they occur, they have long durations and impacts, e.g., sea level rise, or increased prevailing atmospheric temperatures.

These change substantially the required coping strategies from those appropriate to conventional disasters, increasingly emphasising instead the need for anticipating trends, mitigating their likely effects, more permanent evacuation from vulnerable localities, adaptive food security policies and the like. Accordingly, and in contrast to disaster preparedness, longer term behavioural adaptation is recognised as being relatively more important than short- to medium-term mitigation. Politically, however, such lifestyle changes may prove difficult and unpalatable. Hence, despite the current British government's concern with, and claimed prioritisation of, GEC issues, Prime Minister Tony Blair recently went on record as saying that it was unrealistic to expect people to change their way of life, e.g., by reducing their use of cheap flights, for the sake of the environment. Adaptation requires precisely such changes. These will need to go far beyond the marginal or cosmetic energy-saving initiatives such as switching off lights 
in unoccupied rooms, not leaving electronic appliances on standby and reducing car mileage by carefully combining separate single-purpose trips into multipurpose journeys. The wealthy use and waste far more resources than the poor, raising present-day issues of ethics, equity and environmental justice even before intergenerational effects are considered.

\subsection{An urban world}

One key factor distinguishing the present human condition - and its antecedents as far back as the industrial revolutions - is that Homo sapiens has become an urban species. For the first time in human history, half of the world's current population of some 6.6 billion people resides in areas defined as urban. Therefore cities and the activities carried out in them will play a central role in determining our future. The interlinked processes of urbanisation, industrialisation and globalisation are changing lifestyles, resource use and waste production in unprecedented ways, including climate change, that are threatening the sustainability of the biosphere (e.g., O'Brien and Leichenko, 2003; Sánchez-Rodríguez et al., 2005).

In recent years, the most rapid urbanisation has been occurring in Southeast Asia and especially China - where the transformation of Beijing, Shanghai and the mushrooming megacity region of the Pearl River Delta symbolise China's increasing integration into the world-economy and rise as a global power. Even in sub-Saharan Africa, the world's least urbanised continental region overall, several countries are now more than 50\% urbanised, while the likes of Cairo, Kinshasa, Lagos and the Gauteng region centred on Johannesburg have also become teeming megacities ${ }^{1}$ characterised by a familiar set of problems and challenges as well as opportunities. While mega and other large cities tend to dominate the headlines and policy agendas, very often it is secondary and intermediate cities that have been growing most rapidly in recent years (Montgomery et al., 2003). Many cities, particularly under conditions of rapid growth or stagnation, are also highly polarised between rich and poor. The extent to which urban poverty is addressed will determine significantly how well or otherwise the anti-poverty targets of the Millennium Development Goals (MDGs) will be met (UN-Habitat, 2006). We truly live in an urbanised world, and the state of cities is therefore crucial to well-being and quality of life.

\subsection{The state of GEC research}

Growing concern about GEC is attested most notably by the publication in late 2006 of the authoritative Stern Review Report commissioned by the UK Treasury, which focused on the economic implications of climate change. Despite its over 700 page length, the report also surveyed the state of scientific knowledge in fairly accessible form and from where the following data are drawn.

Prior to the industrial revolution, atmospheric $\mathrm{CO}_{2}$ levels averaged 280 parts per million (ppm). Since then, concentrations of greenhouse gases (GHGs) have been rising at an increasing rate, now more than $2 \mathrm{ppm}$ annually. The current level stands at roughly $430 \mathrm{ppm}$ of $\mathrm{CO}_{2}$ equivalent. ${ }^{2}$ Unless decisive action is taken urgently, predictions point to $\mathrm{CO}_{2}$-equivalent concentrations of $550 \mathrm{ppm}$ of being reached between 2035 and 2050. This is likely to increase in mean atmospheric temperatures to $2^{\circ} \mathrm{C}$ above pre-industrial levels, compared with the $0.5^{\circ} \mathrm{C}$ increase experienced to date. By the end of this century, 
unabated emission discharges would raise the levels of $\mathrm{CO}_{2}$ equivalent to around $750 \mathrm{ppm}$, with a $50 \%$ chance of a $5^{\circ} \mathrm{C}$ temperature increase. To underline the seriousness of the problem, the Stern Review points out that this would be the same magnitude of temperature increase as has occurred since the last Ice Age. "Such a radical change in the physical geography of the world must lead to major changes in the human geography - where people live and how they live their lives" (Stern Review on the Economics of Climate Change, 2006, p.vi).

The Stern Report calculates that this would also probably reduce global living standards by some $5 \%-20 \%$. However, the worst impacts of climate change could be reduced substantially if GHG levels were stabilised within the range of $450-550 \mathrm{ppm}$. The longer the delay, the harder and more costly it will become to achieve even the upper part of this stabilisation range.

Because atmospheric changes have cumulative and lagged responses, the effects of what is happening today will be felt increasingly over the coming decades, even if we were able to stabilise emissions at current levels immediately. Hence to stabilise GHG levels within the range of $450-550 \mathrm{ppm} \mathrm{CO}_{2}$ equivalent would require global emission levels by 2050 to be at least $25 \%$ below current levels. Another crucial finding of the Stern Report is that tackling climate change represents not so much of a financial and resource cost as a substantial economic opportunity that could boost technical innovation and overall economic activity.

This conclusion, probably more realistic than the wholly pessimistic scenario, really does help to progress debate by enabling us to focus on appropriate means for addressing the problem, rather than on whether we need to or should do so. The timescales involved in these prognoses and the coping strategies that need to be formulated and implemented to tackle them, raise quite explicitly the issue of intergenerational lags and 'bequests' that impact profoundly on equity for future generations. I return to this below.

\section{Urbanisation and global environmental change: key issues}

Overall, GEC research has been heavily dominated - in terms of funding, effort and outputs - by the natural sciences, focusing on climate change measurement and modelling, and the like. This is the type of research that has underpinned the IPCC and its first three assessment reports. This natural scientific and modelling work is clearly important but social science research is still perceived by some natural scientists as something of a Cinderella, even though the need for, and importance of, it is increasing. The establishment of the International Human Dimensions Programme on GEC (IHDP) by a combination of natural and social scientific organisations in the mid-1990s (see below) provides clear evidence of such changing attitudes; similarly, the IPCC's Fourth Assessment Report, to be published during 2007, will have a social scientific component, including attention to urban challenges. These are the so-called 'human dimensions' of GEC.

\section{The International Human Dimensions Programme on GEC}

To redress this imbalance in research effort, the IHDP was established in 1996. It was sponsored by two key global scientific and social scientific unions, with which it works in partnership, namely, the International Council for Science (ICSU) and 
International Social Science Council (ISSC). Its other key partners are the International Geosphere-Biosphere Programme (IGBP), the International Programme on Biodiversity (DIVERSITAS) and World Climate Research Programme (WCRP), with all of which IHDP forms the Earth System Science Partnership (ESSP).

IHDP is headquartered in Bonn but its various research programmes (known as 'core projects') have their own individual International Project Offices (IPOs) at or close to the institutional base of their respective chairs. The projects are directed by small Scientific Steering Committees, work towards their stated objectives (set out in a peer-reviewed Science Plan when they are commissioned) through establishing networks of associated researchers who are either already working on relevant independent projects or seek to situate planned new projects within the terms of reference of the respective Science Plan.

IHDP's first tranche of 'core projects' focused on land use and cover change, aspects of human security, water/hydrological cycles, industrial transformations, and global carbon stocks and flows. In 2005, a new IHDP 'core project' on Urbanisation and Global Environmental Change (UGEC) was launched to focus attention on the bidirectional interactions between the processes and forces of urbanisation and GEC. For analytical clarity, the issues of concern are divided into four interlocking themes within the Science Plan (Sánchez-Rodríguez et al., 2005), namely:

Theme 1 urban processes that contribute to GEC, e.g., industrial and vehicle emissions that add GHGs to the atmosphere

Theme 2 pathways through which GEC affects the urban system, e.g., heat island effects resulting from the heat absorbing nature of urban roofs, roads and other non-natural surfaces

Theme 3 interactions and responses within the urban system, e.g., vulnerabilities of particular groups in cities and coping strategies of groups and institutions

Theme 4 consequences of interactions within urban systems on GEC, e.g., how different urban coping mechanisms affect GHG emissions and climatic variability, and whether different types of urban area adapt differentially.

Existing urban GEC research has been fragmented and often locality-specific, focusing predominantly on concerns within Themes 1 and 2 . However, the other dimensions are equally important. Theme 4 is arguably the least well understood and the aspect on which least research has been carried out to date. Initial efforts will concentrate on Themes 1-3, with the emphasis on 2 and 3. It is envisaged that this will build up sufficient of a research base to enable Theme 4 to be addressed later in the core project's life (Sánchez-Rodríguez et al., 2005).

The scope of this research programme is global but with a particular emphasis on regional and other systematic and structural differences. For instance, the contribution to GEC of urban areas within parts of the global South is increasing rapidly (most dramatically in countries like China and India). This is of particular concern since the skills, capacity and resources to tackle its impacts, let alone to anticipate change and undertake remedial actions, are often severely lacking. Moreover, some of the most dramatic impacts of GEC will be felt in poor countries and especially by the poorest and most vulnerable elements of society, in both urban and rural areas. Multidisciplinary regional networks of associated researchers are being established to foster collaboration and to address the challenges of making interregional comparisons. ${ }^{3}$ 
Much of the previously existing research on urban impacts and coping mechanisms was undertaken in the context of so-called 'natural' disasters (not least under the auspices of the UN Decade on Natural Disaster Reduction during the 1990s) - see above; there is little explicitly on urbanisation and GEC issues.

\section{GEC, urbanisation and intergenerational equity}

Intergenerational equity issues need to be highlighted and taken into account because of the rapidly increasing levels of urbanisation in parts of the global South, coupled with the long-term nature of the impacts of GEC. This is true BOTH in the short- to medium-term sense of mitigation and coping strategies AND in the context of the inherent long-term nature of sustainable development a là the Brundtland Commission report (WCED, 1987) or many other definitions that emphasise:

- the imperative of present-day development not being at the expense of future generations

- the central importance of poverty reduction/elimination if the concept is to have meaning.

Underpinning this latter are two key issues:

1 A pragmatic realisation that poor people often degrade their environment in their daily struggles to survive through lack of choice rather than ignorance, and an associated moral/ethical concern with social equity and justice. While such problems are perhaps more readily appreciated in rural contexts, urban environments of poverty are equally problematic, e.g., the consequences of urban and peri-urban deforestation or overcrowded slums and shantytowns lacking safe water supply and adequate sanitation and refuse collection services. Traditional, top-down developmentalist approaches that rely on outside 'experts' (whether from within the same country or abroad) telling supposedly unknowing poor indigenes what and how to do have long been discredited but are still common. However, numerous alternative approaches, under the broad rubric of participatory methods, co-evolution, postcolonialism, sustainable livelihoods, political ecology, alternative resource accounting procedures, social cost-benefit analysis and the like, have demonstrated the potential (and difficulties) of working with the intended beneficiaries as equals who hold considerable 'indigenous knowledge' and local experience. Accordingly, the strategy for tackling environmental degradation caused by such groups of people should focus on relieving survival constraints and exploring alternative activities within their control. Failure in this respect will result in accelerating environmental problems and increased poverty, propelling GEC into the realm of a real threat to human security. In O'Brien's (2006, p.1) words, which reflect the approach of the IHDP's GEC and Human Security 'core project'

(GECHS, 1999):

In terms of environmental change, human security can be considered the condition when and where individuals and communities have the options necessary to end, mitigate or adapt to risks to their human, environmental and social rights, have the capacity and freedom to exercise these options; and actively participate in attaining these options. 
2 A concern with environmental justice, both globally but even more dramatically in the global South. From this perspective, issues of environmental rights and opportunities apply both intergenerationally (in keeping with the Brundtland Commission's approach to sustainable development as a long-term process as cited above) and horizontally at a particular point in time. In other words, the activities and resource uses of the wealthy should not impinge on or remove the rights of the poor and less powerful to enjoy their own environmental rights. GECs link these two, since current polluting industrial production or environmental contamination through inadequate sewerage infrastructure, for instance, are likely BOTH to affect particular groups of urban and peri-urban residents today AND to have cumulative impacts in the future.

Rural examples clearly also exist, e.g., deforestation or toxic contamination from mining activities. However, the increasing concentration of non-agricultural activities in burgeoning cities, where over half the world's population now live, many in conditions of absolute and/or relative poverty, means that the challenges of addressing GEC and ensuring the social, economic and environmental sustainability of human development today and in the future are increasingly becoming urban challenges. As pointed out in the summary of current GEC science above, if not tackled effectively, current increases in atmospheric concentrations of GHGs will affect global climate in 40 or 50 years' time even more severely than today. As explained here, environmental justice is actually very close to O'Brien's (2006) concept of human security.

\section{Conclusion}

Urbanisation and GEC are crucially interrelated yet their two-way relationships remain very under-researched. Hence their consequences are still too often inadequately appreciated, in terms either of present-day or long-term impacts. On account of the increasing severity and frequency of extreme events, coupled with the slow-onset yet semi-permanent nature of many of its phenomena, GEC is substantially different from conventional disasters and the associated literature or policy agenda. Ultimately, addressing the GEC challenge requires us to confront new approaches to sustainable development that relate both to intergenerational equity and cross-sectional social equity and environmental justice. Human security also embraces both these time axes. As the geographies of international divisions of labour continue to change in our intensely globalising world, so too will the spatial, economic, environmental and social dimensions of wealth creation and impoverishment. One of the most profound recent changes has been the ongoing urbanisation of humankind, to the point where our habitat is now becoming more urban than rural. Strategic thinking, policy and practice must therefore change accordingly, not just to address challenges of urban inequality and justice today but to ensure sustainability and justice for future generations.

\section{Acknowledgements}

This is a revised and expanded version of the paper presented at the Green Economics Institute Conference on Intergenerational Equity, University of Oxford, 8 April 2006. 


\section{References}

GECHS (1999) Global Environmental Change and Human Security; GECHS Science Plan, Bonn: International Human Dimensions Programme on Global Environmental Change, http://www.ihdp.org.

Montgomery, R., et al. (2003) Cities Transformed; Demographic Change and Its Implications in the Developing World, Division of Behavioural and Social Sciences and Education, National Research Council, Washington, DC: Committee on Population.

O'Brien, K. (2006) 'Are we missing the point? Global environmental change as an issue of human security', Global Environmental Change, Vol. 16, No. 1, pp.1-3.

O'Brien, K. and Leichenko, R. (2003) 'Double exposure: assessing the impact of climate change within the context of economic globalization', Global Environmental Change, Vol. 10, No. 3, pp.221-232.

Sánchez-Rodríguez, R., Seto, K., Simon, D., Solecki, W., Kraas, F. and Laumann, G. (2005) Science Plan: Urbanization and Global Environmental Change, Bonn: International Human Dimensions Programme on Global Environmental Change, p.60, http://www.ihdp.org, www.ugec.org.

Stern Review on the Economics of Climate Change (2006) Final Report, http://www.hm-treasury .gov.uk/independent_reviews/stern_review_economics_climate_change/stern_review_report .cfm (accessed 30 October 2006).

UN-Habitat (2006) State of the World's Cities 2006/7, London: Earthscan.

Vitousek, P. (1992) 'Global environmental change: an introduction', Annual Review of Ecological Systems, Vol. 23, No. 1, pp.1-14.

World Commission on Environment and Development (WCED) (1987) Our Common Future; The Report of the Brundtland Commission, New York: Oxford University Press.

\section{Notes}

1 Definitions of megacities vary but the most widely used, endorsed by UN agencies, uses a minimum population of ten million.

$2 \mathrm{CO}_{2}$ equivalent is a measure of all GHGs converted to their equivalent in $\mathrm{CO}_{2}$ terms for simplicity. The six principal GHGs covered by the Kyoto Protocol are carbon dioxide, methane, nitrous oxide, PFCs, HFCs and SF.

3 See http://www.ugec.org for application procedures. 